\title{
Noncommutative Geometry and Fundamental Interactions: The First Ten Years
}

\author{
José M. Gracia-Bondía \\ Departamento de Física Teórica I, Universidad Complutense, \\ 28040 Madrid, Spain*
}

October 9, 2018

\begin{abstract}
This is the full text of a survey talk for nonspecialists, delivered at the 66th Annual Meeting of the German Physical Society in Leipzig, March 2002. We have not taken pains to suppress the colloquial style. References are given only insofar as they help to underline the points made; this is not a full-blooded survey. The connection between noncommutative field theory and string theory is mentioned, but deemphasized. Contributions to noncommutative geometry made in Germany are emphasized.
\end{abstract}

\section{Mathematical prehistory}

As a branch of mathematics, Noncommutative Geometry (NCG) is 20 years old. It was precisely in Germany, at the Oberwolfach meeting in September-October 1981, that Connes unveiled a "homology of currents for operator algebras" [1] amounting to differential analysis on noncommutative algebras.

Geometric properties of noncommutative algebras had already been worked out in detail for the noncommutative torus, then known as the "irrational rotation $C^{*}$-algebra", by Connes himself [2]; whereupon the canonical trace on the torus algebra gives a "noncommutative integral".

The related calculus, reformulated in the new language of cyclic cohomology, was developed in detail in the foundational paper on "Noncommutative Differential Geometry" 3], which started to circulate in preprint form around Christmas, 1982. A high point of this article is the so-called Hochschild-Kostant-Rosenberg-Connes theorem [4]. By 1983, Connes' approach to cyclic cohomology was essentially complete [5]; his parallel work on foliations and noncommutative integration led him to emphasize the role of Fredholm modules in the NCG formalism. The Baum-Connes and Connes-Kasparov conjectures were formulated in

*Permanent address: Departamento de Física, Universidad de Costa Rica, 2060 San Pedro de Montes de Oca, Costa Rica. 
their primitive form in the early eighties. Also pioneer work by Rieffel [6] contributed to shape noncommutative geometry in its early stages.

Noncommutative geometry, in essence, is an operator algebraic, variational reformulation of the foundations of geometry, extending to noncommutative spaces. NCG allows consideration of "singular spaces", erasing the distinction between the continuous and the discrete. Its main specific tools are Dirac operators, Fredholm modules, the noncommutative integral, Hochschild and cyclic cohomology of algebras, $C^{*}$-modules and Hopf algebras. NCG has many affinities with quantum field theory, and it is not unusual for noncommutative homological constructs to crop up in that context [7].

On the mathematical side, NCG has had a vigorous development. Current topics of interest include index theory and groupoids, mathematical quantization, the Novikov and Baum-Connes conjectures and the relation of the latter to the Langlands program, locally compact quantum groups, the dressing of fermion propagators in the framework of spectral triples, and the famous Riemann hypothesis. In the mainstream of mathematics, the noncommutative program needs no tribute. It is here to stay.

\section{The Interface with Physics}

We say interface because indeed, there is no question of "application" of NCG to physics, but rather of mutual intercourse. In fact, the original use of noncommutative geometry in physics was a model of epistemological humility. Instead of trying to derive the laws of physics from some NCG construct, people were trying to learn, from the mainstream physical theories, what the noncommutative geometry of the world could be. By "mainstream physical theories" we essentially understand the Standard Model (SM) of fundamental interactions.

The Standard Model, which has been been termed "ugly" by so many physicists, including some of its fathers, is dramatically elegant and beautiful in modern mathematics, in that its crucial concepts are among the deepest and most powerful in noncommutative geometry. I am talking about the concepts of gauge field and of chiral fermion.

Gauge fields are identical with connections, perhaps the most important objects in the modern formulation of geometry. The algebraic definition of linear connection is imported verbatim into NCG. Thinking seriously about the space of all connections has been a very fruitful idea that mathematicians have picked up from physicists [8].

Chiral fermions are acted on by Dirac and Dirac-Weyl operators. Perhaps it is not so well known that Dirac operators are a source of NCG, where they give rise to "fundamental classes". (Any complex spinor bundle on a smooth manifold gives rise to a generalized Dirac operator $D$, whose sign operator $F=D|D|^{-1}$ determines a Fredholm module; the natural equivalence class of these depends only on the underlying $\operatorname{spin}^{c}$-structure [9]. Since the $\operatorname{spin}^{c}$ structure determines, among other things, the orientation of the manifold, this class is a finer invariant than the usual fundamental class in homology.) It is precisely the role of the Dirac operator both in the Standard Model and in NCG that made the rapprochement between this branch of mathematics and fundamental physics natural and unavoidable.

There have been two main lines for making sense of the SM in terms of NCG: the one developed by Connes himself (and followers), and the one related to the Lie superalgebra 
$\mathfrak{s u}(2 \mid 1)$, mainly exploited by the Mainz-Marseille group (Scheck, Häußling, Paschke, Papadopoulos, Coquereaux, Esposito-Farèse, Vaillant), more or less at the same time.

Let us collect the gauge potentials appearing in the SM into a single package of differential forms:

$$
\mathbb{A}^{\prime}=i(B, W, A)
$$

where

$$
B=-\frac{i}{2} g_{1} \mathbf{B}_{\mu} d x^{\mu}, \quad W=-\frac{i}{2} g_{2} \boldsymbol{\tau} \cdot \mathbf{W}_{\mu} d x^{\mu} \quad \text { and } \quad A=-\frac{i}{2} g_{3} \boldsymbol{\lambda} \cdot \mathbf{A}_{\mu} d x^{\mu},
$$

with $\mathbf{B}, \mathbf{W}$ and $\mathbf{A}$ denoting respectively the hypercharge, weak isospin and colour gauge potentials. From the mathematical standpoint, $W$ is to be regarded as a 1-form with values in the real field $\mathbb{H}$ of quaternions. In other words, $\mathbb{A}^{\prime}$ is an element of $\Lambda^{1}(M) \otimes \mathcal{A}_{F}$, where the noncommutative algebra $\mathcal{A}_{F}:=\mathbb{C} \oplus \mathbb{H} \oplus M_{3}(\mathbb{C})$, that we baptized the Eigenschaften algebra [10, plays the crucial rôle.

Let us also collect all chiral fermion fields into a multiplet $\Psi$ and denote by $J$ the charge conjugation operation. Then the fermion kinetic term is rewritten as follows:

$$
I\left(\Psi, \mathbb{A}^{\prime}, J\right)=\left\langle\Psi \mid\left(i \not \partial+\mathbb{A}^{\prime}+J \mathbb{A}^{\prime} J^{\dagger}\right) \Psi\right\rangle .
$$

Next we look at the Yukawa part of the SM Lagrangian. Use the normalized Higgs doublet $\Phi:=\left(\begin{array}{l}\Phi_{1} \\ \Phi_{2}\end{array}\right):=\sqrt{2} \phi / v$, where $\phi$ is a Higgs doublet with vacuum expectation value $v / \sqrt{2}$. We also need

$$
\widetilde{\Phi}:=\left(\begin{array}{c}
-\bar{\Phi}_{2} \\
\bar{\Phi}_{1}
\end{array}\right)=\left(\begin{array}{cc}
0 & -1 \\
1 & 0
\end{array}\right)\left(\begin{array}{l}
\bar{\Phi}_{1} \\
\bar{\Phi}_{2}
\end{array}\right) .
$$

It has been remarked that the Higgs should also be properly regarded as a quaternion-valued field; this makes apparent the custodial symmetry of the scalar sector of the SM, which is related to the $\rho=1$ tree level prediction for the low-energy neutral to charged current interactions ratio.

Introduce then $q_{\Phi}=\left(\begin{array}{cc}\bar{\Phi}_{1} & \bar{\Phi}_{2} \\ -\Phi_{2} & \Phi_{1}\end{array}\right)$ and $r:=q_{\Phi}-\left\langle q_{\Phi}\right\rangle=q_{\Phi}-1$ and write, schematically for a right-left splitting of the fermion multiplets:

$$
\mathbb{A}^{\prime \prime}=\left(\begin{array}{cc}
r^{\dagger} M &
\end{array}\right)
$$

where $M$ denotes the quark or lepton mass matrix (including the Kobayashi-Maskawa or Maki-Nakagawa-Sakata parameters), as the case might be. Just as the standard Dirac operator relates the left- and right-handed spinor representations, the Yukawa operator $\mathcal{D}_{F}$ in the space of internal degrees of freedom relates also the left- and right-handed chiral sectors. The Yukawa terms for both particles and antiparticles (for the first generation) are written

$$
\begin{aligned}
I\left(\Psi, \mathbb{A}^{\prime \prime}, J\right):=\left\langle\Psi \mid\left(\mathcal{D}_{F}+\mathbb{A}^{\prime \prime}+J \mathbb{A}^{\prime \prime} J^{\dagger}\right) \Psi\right\rangle \\
=\bar{q}_{L} \Phi m_{d} d_{R}+\bar{q}_{L} \widetilde{\Phi} m_{u} u_{R}+q_{R} \bar{\Phi} \bar{m}_{d} \bar{d}_{L}+q_{R} \widetilde{\bar{\Phi}} \bar{m}_{u} \bar{u}_{L} \\
\quad \quad \quad \bar{\ell}_{L} \Phi m_{e} e_{R}+\bar{\ell}_{L} \widetilde{\Phi} m_{\nu} \nu_{R}+\ell_{R} \bar{\Phi} \bar{m}_{e} \bar{e}_{L}+\ell_{R} \widetilde{\bar{\Phi}} \bar{m}_{\nu} \bar{\nu}_{L}+\text { h.c. }
\end{aligned}
$$


Note that neutrinos are assumed to have only Dirac mass terms, and that the hypercharges of $\Phi, \widetilde{\Phi}$ are respectively +1 and -1 .

The combination of both constructions yields a Dirac-Yukawa operator $\mathcal{D}=i \not \partial \oplus \mathcal{D}_{F}$. With $\mathbb{A}:=\mathbb{A}^{\prime} \oplus \mathbb{A}^{\prime \prime}$, the whole fermionic sector of the SM being recast in a form similar to (11):

$$
I(\Psi, \mathbb{A}, J)=\left\langle\Psi \mid\left(\mathcal{D}+\mathbb{A}+J \mathbb{A} J^{\dagger}\right) \Psi\right\rangle .
$$

In keeping with the old Kaluza-Klein idea, we have combined the ordinary gauge fields and the Higgs as entries of a generalized gauge field. The Yukawa terms come from the minimal coupling recipe applied to the gauge field in the internal space. Hence the "twoleaves" picture for the Glashow-Weinberg-Salam model [11, 12] that contributed to make intuitive the noncommutative vision of the SM. The Dirac-Yukawa operator is seen to contain in NCG all the relevant information pertaining to the SM.

The construction was made in the realm of Euclidean field theory. I believe Mario Paschke will explain to us in this Meeting the (difficulties and) hopes for introducing time in the picture.

This Connes-Lott reconstruction of the SM gave rise to two "predictions". It is important to recall that, at the time they were made, the top quark had not yet been seen, and the best estimates for its mass clustered around $130 \mathrm{GeV}$ ! The NCG model sort of explains why the masses of the top quark, the $W$ and $Z$ particles and the Higgs particle should be of the same order, and gave right away

$$
m_{\mathrm{top}} \geq \sqrt{3} m_{W} \approx 139 \mathrm{GeV} .
$$

With a bit of renormalization group running [13], it fell right on the mark.

On the other hand, the "prediction" for the Higgs mass from Connes' NCG has remained stubbornly around $200 \mathrm{GeV}$, which is too large for current phenomenological prejudice.

The Mainz $\mathfrak{s u}(2 \mid 1)$ model was based on the following idea: put together the $(W, B)$ forms belonging to the $\mathfrak{s u}(2) \times \mathfrak{u}(1)$ gauge group into a $3 \times 3$ antihermitian matrix and fill up the lateral columns with the Higgs field components:

$$
M=\left(\begin{array}{ll}
W_{2 \times 2} & C_{2 \times 1} \\
D_{1 \times 2} & B_{1 \times 1}
\end{array}\right)_{3 \times 3},
$$

with

$$
\operatorname{Str} M=\operatorname{tr} W-B=0 .
$$

This gives rise to the Lie superalgebra, and then we can look for its representations and relate them to the fermion fields. The idea goes back to [14, but its more modern proposers realized that the superalgebra should not be gauged.

It turns out that the Mainz model gives as good a description as the Connes-Lott machine of spontaneous symmetry breaking, and that the known families of quarks and leptons fit very well with the (reducible but indecomposable) $\mathfrak{s u}(2 \mid 1)$ representations [15.

This model received less attention than Connes', perhaps because the Dirac operator does not play the main role. However, the big prize nowadays, the true pot of gold, lies in "horizontal symmetry"; all the more so since we have come to realize that the neutrino 
sector has a much richer structure which was found a few years ago. The attraction of the Mainz model, or variants thereof, over Connes', is that it gives some clues to the structure of the fermion mass matrix; and so, that model is alive and well.

On this score, I offer a decidedly low-tech suggestion. Let us start from the same idea of completing the $\mathfrak{s u}(2) \times \mathfrak{u}(1)$ into a $3 \times 3$ matrix. Go back to the ordinary Lie algebra context in mixing the four Higgs field components with a $\mathfrak{u}(3)$ symmetry (it has always been clear that $\mathfrak{s u}(3)$ won't work). The extra scalar field comes in handy for astrophysical purposes.

In other words, we recognize the basic fact from covariant wave equations theory, and from the very structure of the SM that spin 0 and spin 1 fields always appear in tandem. The exercise gives the right quantum numbers. The Lagrangian for the Glashow-WeinbergSalam model, except for the symmetry-breaking terms, is elegantly rewritten in terms of the Dirac operator only [16]. An interesting fact is that, with the appropriate variables, the Lagrangian for the gravity field can be written in similar form. Now, the "dualized standard models" try to explain the existence of three generations and the relations among them from a duality (effectively taking place at the 100-1000 TeV scales) between the $\mathfrak{s u}(3)$ color sector of the SM and the fermion families space. This scheme is open to criticism in that it is unclear why the leptons, which do not see color, should see dualized color. But the NCG approach taught us that there is a third 3 in the Standard Model. So, just maybe, there is a triality lurking behind it.

Apart from the above (which is mostly old hat), we would say that the first stage of the process of linking NCG and fundamental physics finished around the winter of 1995-96. The humble approach paid handsomely up, when the very structure of the SM itself gave Connes the inspiration for the construction of noncommutative spin manifolds. That is, since that time we know how to put fermion fields on a noncommutative manifold, and this knowledge is backed by a fundamental mathematical result, whose proof you can find in Elements of Noncommutative Geometry 4]. This needs in turn the proof of the "character theorem", stated but not proved in [17, which was provided to us by Connes.

Afterwards, there has been little activity on this front. The vexing question of the unimodularity condition, which has to be imposed on the noncommutative gauge field (2) in order to really reproduce the Standard Model with the correct hypercharges, was "explained" by Alvarez, Martín and myself in terms of anomaly cancellation [18; but not understood on a native NCG basis. This loose thread has been tied up recently by Serge Lazzarini and Thomas Schücker [19].

\section{Arrival of the Top-down Approach}

We have seen that the first cohabitation, in recent times, of noncommutative geometry and the physics of fundamental interactions actually followed a bottom-up pattern. The top-down approach goes back to the classic paper by Snyder in January 1947, "Quantized space-time" [20].

In this charming paper, it was first suggested that coordinates are noncommuting operators; the six commutators of the coordinates are proportional to a basic unit of length and correspond to the infinitesimal generators of the Lorentz group; throughout, Lorentz covariance is maintained. Then as now, motivations for using noncommuting coordinates to 
describe spacetime were the hope of improving the renormalizability of QFT and of grappling with the attendant nonlocality of physics at the Planck scale (uncertainty relations for spacetime coming from gravity).

The top-down approach has been resurrected recently by string theorists. In their most popular model, the commutation relations are simply of the form

$$
\left[x^{j}, x^{k}\right]=i \theta^{j k}
$$

breaking Lorentz invariance. As anticipated by Sheikh-Jabbari [21] and from a slightly different viewpoint by Schomerus [22], and plausibly argued by Seiberg and Witten [23], open strings with allowed endpoints on 2Dirichlet-branes in a $B$-field background act as electric dipoles of the abelian gauge field of the brane; the endpoints live on the noncommutative space determined by (3). Then Kar [24] argued that the holographic correspondence relates the noncommutative and commutative worlds.

Slightly before, Douglas, Schwarz and Connes himself [25] had arrived at the conclusion that compactification of $M$-theory, in the context of dimensionally reduced gauge theory actions, leads ineluctably to noncommutative spaces. And Douglas and Hull [26] had inferred from this that gauge theories on NC tori make sense as a limit in string theory. Other work relating noncommutative geometry and strings includes [27], partly inspired by [28].

It should also be noted that Doplicher, Fredenhagen and Roberts had earlier considered a model with commutation relations

$$
\left[x^{j}, x^{k}\right]=i \sigma^{j k}
$$

where the $\sigma^{j k}$ are the components of a tensor, and beyond that are $c$-numbers for all practical purposes. Thus in their formalism [29, Lorentz invariance is also explicitly kept.

A very important construction in the framework of [23] is the so-called Seiberg-Witten map in gauge theory. This map relates the gauge fields and the gauge variations in a noncommutative theory with commutative counterparts. Let $\widehat{A}(A)$ and $\hat{\lambda}(A, \lambda)$, where

$$
\hat{\delta} \widehat{A}_{j}=\partial_{j} \hat{\lambda}+i\left(\widehat{A}_{j} \star_{\theta} \hat{\lambda}-\hat{\lambda} \star_{\theta} \widehat{A}_{j}\right)
$$

be, respectively, the $\mathrm{NC}$ gauge potentials and gauge variation in terms of the commutative ones; the $\star_{\theta}$ denotes the Moyal product, of which more below. Then necessarily, in order to have

$$
\widehat{A}(A)+\hat{\delta} \widehat{A}(A)=\widehat{A}(A+\delta A)
$$

for $\theta$ small,

$$
\begin{aligned}
\widehat{A}_{j}(A) & =A_{j}-\frac{1}{4} \theta^{k l}\left\{A_{k}, \partial_{l} A_{j}+F_{l j}\right\}+O\left(\theta^{2}\right), \\
\hat{\lambda}(A, \lambda) & =\lambda+\frac{1}{4} \theta^{k l}\left\{\partial_{k} \lambda, A_{l}\right\}+O\left(\theta^{2}\right),
\end{aligned}
$$

where $F(A)$ is the ordinary gauge field for $A$. These equations have been seen by Jurčo, Schupp and Wess 30, and also by Jackiw and Pi 31, to correspond to an infinitesimal 1-cocycle for a projective representation of the underlying gauge group in the Moyal algebra.

The equations (5) are valid for arbitrary $\theta$, and so, when using the Seiberg-Witten map, the components of $\theta$ should be regarded as variables. 
Before continuing, we need an interlude on the Moyal product. It is correctly (nonperturbatively) defined, for nondegenerate $\theta$, as

$$
f \star_{\theta} g(u):=(\pi \theta)^{-4} \int_{\mathbb{R}^{4}} \int_{\mathbb{R}^{4}} f(u+s) g(u+t) e^{2 i s \theta^{-1} t} d s d t,
$$

and this gives rise to the commutation relations (3).

Mathematically, those are the commutation relations of Quantum Mechanics, when the reduced Planck constant replaces $\theta$ ! From now on, we neglect to indicate $\theta$ in the star product notation. The precise relation of (6) to the asymptotic formula usually given as definition of the Moyal product was spelled out some time ago in [32. Indeed the formula (6) is the basis of the "fourth" formalism of Quantum Mechanics, in which observables, states and transitions are described by functions (or distributions) on phase space. This formalism, which goes back to Wigner, Weyl and Moyal, had already a long and proud history when (a version of) the Moyal product was rediscovered by string theorists. And so, as Fedele

Lizzi has put it, "in the string community we can happily go on rediscovering quantum mechanics" 33 .

An even more fortunate fact was that the quantum field-theoretical framework for making sense of the noncommutative limit of string theory also preexisted the paper by Seiberg and Witten. This story deserves its own chapter.

\section{Noncommutative Field Theory (NCFT)}

NCFT can be and was developed independently of its string theory motivation and background. I go not into the theory of quantum fields on ad-hoc discrete spaces (in this Conference, Rainer Häußling will touch on that). In turn the latter topic should not be confused with "fuzzy physics", which has led nowhere, and does deserve a sharp look - but not here. A reasonably good review for NCFT is [34].

Let us explain why NC field theory preexisted the Seiberg-Witten paper. Connes' noncommutative spin manifold theorem and Fredholm module theory, referred to above, come in handy here. Quantum field theory has an algebraic core independent of the nature of space-time. From the representation theory of the infinite dimensional orthogonal group, or an appropriate sugbroup thereof, with the input of a single-particle space, it is possible to derive all Fock space quantities of interest. Nothing really changes if the "matter field" evolves on a noncommutative space. In a nutshell: one can apply the canonical quantization machinery to a noncommutative kind of single-particle space [35].

We already mentioned the long-standing hope that giving up locality in the interaction of fields - one of the basic tenets of quantum field theory and indeed one of the main selling points by the forefathers - would be rewarded with a better ultraviolet behaviour. This hope was now amenable to rigorous scrutiny, and it is not borne out. QFT on noncommutative manifolds also requires renormalization. This, in some sense the first result of NCFT, was proved in general by Várilly and myself in [35. A subsequent important paper by Chaichian, Demichev and Prešnajder, written in a different spirit, confirmed it [36].

Of course, one can prove the same in the context of a particular NCG model, by writing down the integral corresponding to a Feynman diagram, and finding it to be divergent. 
That had been shown previously by Filk [37, for the scalar Lagrangian theory associated the Moyal product algebra. (Filk had been active in "Twisted Eguchi-Kawai" models, and retrospectively it seems clear that he took his cue from [38], where these models were generalized to continuous momenta.)

Two other pioneering papers which predated the one by Seiberg and Witten were [39] and [40. These are important because they show that, at the one-loop level, the radiative corrections respect the gauge (BRS) invariance of the theory. This is not trivial in noncommutative Yang-Mills theory: for instance, it involves curious cancellations for the 4-point function.

Before enumerating the main issues in NCFT, let us issue a warning. There has been an enormous outpouring of literature concerning those issues. The quality of many of those papers is substandard; they should come with an attached notice of Warnung. To begin with, the mathematics of the Moyal product, as already said, antedates its current use by string theorists. There has been no shortage of mathematical mistakes, attesting that many people did not bother to study the Weyl-Wigner-Moyal formalism in depth. For instance, in [41] we find the following assertion (unchallenged till now, to our knowledge):

Let $S S^{m}$ be the set of functions on phase space defined by the condition $S S^{m}=\left\{f(q, p):\left|\partial_{q}^{\alpha} \partial_{p}^{\beta} f\right| \leq C_{\alpha \beta}\left(1+q^{2}+p^{2}\right)^{(m-\alpha-\beta) / 2}\right\}$, for constants $C_{\alpha \beta}$. Then one can show that $f$ being in $S S^{m}$ with $m \leq 0$ implies that $\hat{O}_{f} \in \mathcal{B}(\mathcal{H})$ while $m<0$ implies that $\hat{O}_{f} \in \mathcal{K}(\mathcal{H})$. Thus we see that the Weyl transforms of bounded operators are bounded functions [...]

The last phrase is a non sequitur. It is moreover incorrect. For instance, the (inverse) Weyl transform of the parity operator, which is obviously bounded, is a delta function [42]. In general, the Fourier transform of a function giving a bounded operator gives a bounded operator, too, although perhaps for a different value of $\theta$. The correct approach has been in the literature at least since [43, 44]. Also, some papers read as if they were conceived by randomly opening a book on QFT, and trying to make the noncommutative analog of the section which appeared. Particularly obnoxious is the pest of trivial observations, obvious to anyone steeped in noncommutative mathematics, being republished again and again. For example, the note [4] just rehashes a remark in the (substantial) paper [46]. The whole field shows the traces of having grown too quickly, tending to obscure the lasting contributions.

What then are the main issues in NCFT?

- The distinction between planar and nonplanar Feynman diagrams.

Envisage, for instance, the theory given by the action functional

$$
S=\int d^{4} x\left(\frac{1}{2} \frac{\partial \phi}{\partial x^{\mu}} \frac{\partial \phi}{\partial x_{\mu}}+\frac{1}{2} m^{2} \phi^{2}+\frac{g}{4 !} \phi \star \phi \star \phi \star \phi\right)
$$

Generating the Feynman rules like in the commutative theory, the propagators do not change, but the vertices get in momentum space a factor proportional to

$$
\exp \left(-i / 2 \sum_{1 \leq k<l \leq 4} p_{k \alpha} \theta_{\alpha \beta} p_{l \beta}\right) ;
$$


we suppose all the momenta are incoming on the vertex, in cyclic order.

Consider then a simple diagram of the model like the tadpole diagram; let $p$ denote the incoming momentum and $k$ the loop variable. Depending on the order of the momenta, the previous factor is equal to 1 or to $e^{-i p_{\alpha} \theta_{\alpha \beta} k_{\beta}}$.

In general, planar diagrams are those that get overall phase factors depending only on the external legs; for nonplanar diagrams there are phase factors which depend on loop variables as well, and the corresponding integrals become convergent. For the tadpole diagram, we get amplitudes of the form

$$
\Gamma_{\mathrm{pl}}(p) \propto \int \frac{d^{4} k}{k^{2}+m^{2}}, \quad \Gamma_{\mathrm{npl}}(p) \propto \int \frac{d^{4} k}{k^{2}+m^{2}} e^{-i p \theta k} .
$$

The second integral is finite. Actually, the combinatorics of the nonlocal vertex factors was worked out long ago by González-Arroyo and Okawa in the context of the TEK models, and given in detail in [47.

For noncommutative Yang-Mills theory at the one-loop level, have a look at [4].

- The UV/IR mixing and supersymmetry.

Nonplanar diagrams may become divergent again for particular values of the momenta. This would not seem to matter as long as $\Gamma_{\text {npl }}(p)$ remains a well defined distribution. But, for more complicated diagrams with subdivergences, the dependence on $p$ of their amplitude behaviour spells trouble, because these diagrams may unexpectedly become divergent again. This is the notorious UV/IR mixing, which tends to spoil renormalizability. One can think it reflects the underlying "stringy degrees of freedom". For Moyal noncommutative Yang-Mills theory, this happens already at the 2-loop level. People have tried to get around the obstacle using resummation techniques, or nonlocal field redefinitions, but none of those are convincing to this reviewer so far.

Supersymmetric theories have advantages in regard to renormalizability and the UV/IR trouble 49. In this context, we remark it has been proved by Paban, Sethi and Stern [50] that the deformation leading to NC Yang-Mills theory in the sense of Seiberg and Witten is about the only one compatible with supersymmetry.

- Renormalizability in the context of the Seiberg-Witten map.

Another possibility in order to circumvent the UV/IR problem and obtain renormalizable theories is to use the Seiberg-Witten map. The trouble with this approach is that many new vertices appear, at different orders in $\theta$ and $\hbar$. Extensive calculations [51] have shown that in $\theta$-expanded QED, many miraculous cancellations occur. However, the fermion 4-point function becomes divergent, so, strictly speaking, that model is nonrenormalizable.

Since in this Conference Raimar Wulkenhaar talks about the subject, I shall not spend more time on these matters.

- The question of non/unitarity of theories on spaces with timelike noncommutativity. 
It has been asserted [52] that theories with timelike noncommutativity suffer from violation of unitarity. The issue was lucidly discussed in [53]. However, Dorothea Bahns et al 54] have cogently argued that, when using a Hamiltonian approach to NCFT, instead of the usual Lagrangian approach, this problem can be exorcised. The change in viewpoint concerns only the nonplanar diagrams.

(As we were preparing the manuscript for publication, the idea by Bahns et al has been further elaborated by [55] and [56. We look forward to hear more on this.)

In this context, investigation of the old model by Snyder, unjustly neglected in our opinion, is probably worthwhile. The Hamiltonian approach, nevertheless, probably does not help with UV/IR trouble [57].

- The construction of gauge-covariant/invariant observables.

It will have been clear to the listener that the space coordinates themselves are not gauge-covariant. On the other hand, $x^{\mu}+\theta^{\mu \nu} A_{\nu}$ is a gauge-covariant quantity - as was well known to the practitioners of Weyl-Wigner-Moyal theory. This noncovariance of the space coordinates brings NC theories closer with gravity. The construction of gauge-invariant observables has been competently taken up by Harald Dorn and coworkers in a series of papers [58].

- Anomalies in NCFT.

The part of the nonabelian anomaly which is quadratic in the gauge potentials was found by Carmelo P. Martín and myself [59] to be

$$
\begin{aligned}
& -\frac{1}{96 \pi^{2}} \int d^{4} x \varepsilon_{\mu_{1} \mu_{2} \mu_{3} \mu_{4}} \operatorname{Tr} T^{a}\left[T^{b}, T^{c}\right] \partial_{\mu_{1}} \theta^{a}\left[\widehat{A}_{\mu_{2}}^{b} \star \partial_{\mu_{3}} \widehat{A}_{\mu_{4}}^{c}-\partial_{\mu_{3}} \widehat{A}_{\mu_{4}}^{c} \star \widehat{A}_{\mu_{2}}^{b}\right] \\
& -\frac{1}{96 \pi^{2}} \int d^{4} x \varepsilon_{\mu_{1} \mu_{2} \mu_{3} \mu_{4}} \operatorname{Tr} T^{a}\left\{T^{b}, T^{c}\right\} \partial_{\mu_{1}} \theta^{a}\left[\widehat{A}_{\mu_{2}}^{b} \star \partial_{\mu_{3}} \widehat{A}_{\mu_{4}}^{c}+\partial_{\mu_{3}} \widehat{A}_{\mu_{4}}^{c} \star \widehat{A}_{\mu_{2}}^{b}\right] .
\end{aligned}
$$

The first term of this innocent-looking formula is a new contribution that does not vanish in the noncommutative case. It makes it quite difficult, albeit not impossible [60], to construct nonanomalous chiral theories in NCG.

Further work by C. P. Martín [61] has established that the anomalies are related to the (divergent) planar diagrams: nonplanar diagrams would not break the Ward identities. This last contention, however, has been called in question by [62].

In summary, NCFT offers a brand-new laboratory for QFT. However, when eating this fish, one should beware of the fishbones.

\section{Phenomenological Window}

A game one can now play is to marry the Connes-Lott approach to the new commutation relations. That is to say, to work with the tensor product of (some variant of) the Eigenschaften algebra and a spacetime algebra which is no longer commutative. This has been 
done, for instance, in the following papers: 63], by K. Morita; 64, by Chaichian et al; and, perturbatively in $\theta$, in [65], by the München group. These models should be checked for anomalies.

Noncommutative spacetime coordinates lead to signatures in several $2 \rightarrow 2$ QED treelevel processes in $e^{+} e^{-}$collisions which are in principle observable. In particular, corrections to the amplitudes for pair annihilation, Møller and Bhabha scattering, as well as $\gamma \gamma \rightarrow \gamma \gamma$ scattering, for noncommutative scales $\Lambda_{\mathrm{NC}}$ of order $\geq 1 \mathrm{TeV}$, have been calculated this way and could in principle be probed at linear colliders [66. (Here, $\Lambda_{\mathrm{NC}}$ is defined as $1 / \sqrt{\theta}$, where $\theta$ is a typical parameter in the Moyal product algebra.) Naturally, the wisdom of these calculations, in absence of a proper interpretation of the model at the 2-loop level, is somewhat questionable.

There have also been discussions on the signature of noncommutativity in CP violation phenomena, and the muon magnetic moment "anomaly".

The cosmological implications of a nonzero noncommutativity parameter are also open to discussion. Some people [67, 68] feel that it will modify the quantum perturbations of the inflaton field; it does not seem that noncommutativity can do away with inflation altogether.

\section{Spectral Action and Related Developments}

Meanwhile, Alain Connes has not remained idle. Dissatisfaction with the absence of gravity in the old Connes-Lott model led him to develop the spectral action principle. Together with Chamseddine [69, he proposed a universal formula for an action associated with a (compact) noncommutative spin geometry. The action is based on the spectrum of the Dirac operator and is a geometric invariant. Automorphisms of the manifold underlying the geometry combine both diffeomorphisms and internal symmetries.

The Yang-Mills action functional is there replaced by a "universal" bosonic functional of the form:

$$
B_{\phi}[D]=\operatorname{Tr} \phi\left(D^{2}\right)
$$

with $\phi$ being an "arbitrary" positive function of the Dirac operator $D$. Chamseddine and Connes argue that $B_{\phi}$ has the following asymptotic development:

$$
B_{\phi}[D / \Lambda] \sim \sum_{n=0}^{\infty} f_{n} \Lambda^{4-2 n} a_{n}\left(D^{2}\right) \quad \text { as } \Lambda \rightarrow \infty
$$

where the $a_{n}$ are the coefficients of the heat kernel expansion for $D^{2}$ and $f_{0}=\int_{0}^{\infty} x \phi(x) d x$, $f_{1}=\int_{0}^{\infty} \phi(x) d x, f_{2}=\phi(0), f_{3}=-\phi^{\prime}(0)$, and so on. This was put on a sounder mathematical footing in 70.

They proceed to compute the development for the Dirac-Yukawa operator associated to the Standard Model, obtaining all terms in the bosonic part of the action for the Standard Model, plus unavoidable gravity couplings. That is to say, the spectral action for the Standard Model unifies with gravity at a very high energy scale. Later, a variant of the spectral action was suggested in [71]. 
Wulkenhaar has conjectured that the spectral action for the Moyal algebra has the necessary additional symmetries for gauge theories on $\theta$-deformed spacetime to become renormalizable. To check this, one needs to extend the spectral action to the noncompact NC manifold case; this is indeed one of the burning questions of the hour [72.

Somewhat related to the above, Connes [73] and also Landi [74, Várilly [75], Sitarz [76] and Dubois-Violette [77, have been working on systematically obtaining new noncommutative spaces. One motivation for that is to do quantum gravity in the noncommutative Euclidean context. For that, commutative and noncommutative manifolds alike should be "counted" somehow. The difficulties to count 4-manifolds in the context for quantum gravity are well known - see the magisterial account by E. Alvarez in [78]. Algebraic $K$-theory maybe offers a tool to bypass the problem. Note that one should consider "virtual" NC manifolds; fulfilment of the Hochschild condition [79] might be the criterion to distinguish true classical NC manifolds from virtual ones [80].

\section{On the Connes-Kreimer Hopf Algebra}

Bogoliubov's combinatorial renormalization scheme in dimensional regularization can be summarized as follows. If $\Gamma$ is $1 \mathrm{PI}$ and is primitive (i.e., it has no subdivergences), set

$$
C(\Gamma):=-T(f(\Gamma)), \quad \text { and then } \quad R(\Gamma):=f(\Gamma)+C(\Gamma)
$$

where $C(\Gamma)$ is the counterterm and $R(\Gamma)$ is the desired finite value: in other words, for primitive graphs one simply removes the pole part. Next, we may recursively define Bogoliubov's $\bar{R}$-operation by setting

$$
\bar{R}(\Gamma)=f(\Gamma)+\sum_{\emptyset \subsetneq \gamma \subsetneq \Gamma} C(\gamma) f(\Gamma / \gamma),
$$

with the proviso that

$$
C\left(\gamma_{1} \ldots \gamma_{r}\right):=C\left(\gamma_{1}\right) \ldots C\left(\gamma_{r}\right)
$$

whenever $\gamma=\gamma_{1} \ldots \gamma_{r}$ is a disjoint union of several components. The final result is obtained by removing the pole part of the previous expression: $C(\Gamma):=-T(\bar{R}(\Gamma))$ and $R(\Gamma):=$ $\bar{R}(\Gamma)+C(\Gamma)$. In summary,

$$
\begin{aligned}
& C(\Gamma):=-T\left[f(\Gamma)+\sum_{\emptyset \subsetneq \gamma \subsetneq \Gamma} C(\gamma) f(\Gamma / \gamma)\right], \\
& R(\Gamma):=f(\Gamma)+C(\Gamma)+\sum_{\emptyset \subsetneq \gamma \subsetneq \Gamma} C(\gamma) f(\Gamma / \gamma) .
\end{aligned}
$$

Let now $\Phi$ stand for any particular QFT. The Hopf algebra $H_{\Phi}$ is a commutative algebra generated by one-particle irreducible graphs: that is, connected graphs with at least two

vertices which cannot be disconnected by removing a single line. The product $\Gamma_{1} \Gamma_{2}$ means the disjoint union of the graphs $\Gamma_{1}$ and $\Gamma_{2}$. The counit is given by $\varepsilon(\Gamma):=0$ on any generator, 
with $\varepsilon(\emptyset):=1$ (we assign the empty graph to the identity element). The coproduct $\Delta$ is given, on any $1 \mathrm{PI}$ graph $\Gamma$, by

$$
\Delta \Gamma:=\sum_{\emptyset \subseteq \gamma \subseteq \Gamma} \gamma \otimes \Gamma / \gamma
$$

where the sum ranges over all subgraphs which are divergent and proper (in the sense that removing one internal line cannot increase the number of its connected components); $\gamma$ may be either connected or a disjoint union of several connected pieces. The terms for $\gamma=\emptyset$ and $\gamma=\Gamma$ in the sum are $\Gamma \otimes 1+1 \otimes \Gamma$. The notation $\Gamma / \gamma$ denotes the (connected, 1PI) graph obtained from $\Gamma$ by replacing each component of $\gamma$ by a single vertex.

To see that $\Delta$ is coassociative, we observe that if $\gamma \subseteq \gamma^{\prime} \subseteq \Gamma$, then $\gamma^{\prime} / \gamma$ can be regarded as a subgraph of $\Gamma / \gamma$; moreover, it is obvious that $(\Gamma / \gamma) /\left(\gamma^{\prime} / \gamma\right) \simeq \Gamma / \gamma^{\prime}$. The desired relation $(\Delta \otimes \mathrm{id})(\Delta \Gamma)=(\mathrm{id} \otimes \Delta)(\Delta \Gamma)$ can now be expressed as

$$
\sum_{\emptyset \subseteq \gamma \subseteq \gamma^{\prime} \subseteq \Gamma} \gamma \otimes \gamma^{\prime} / \gamma \otimes \Gamma / \gamma^{\prime}=\sum_{\emptyset \subseteq \gamma \subseteq \Gamma, \emptyset \subseteq \gamma^{\prime \prime} \subseteq \Gamma / \gamma} \gamma \otimes \gamma^{\prime \prime} \otimes(\Gamma / \gamma) / \gamma^{\prime \prime},
$$

which is easy to verify directly.

Here (Figures 14) are some coproducts for $\varphi_{4}^{4}$ diagrams:

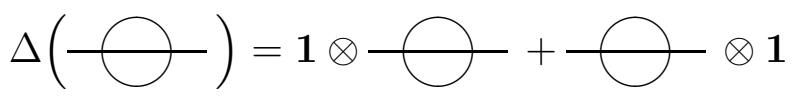

Figure 1: The "setting sun": a primitive diagram

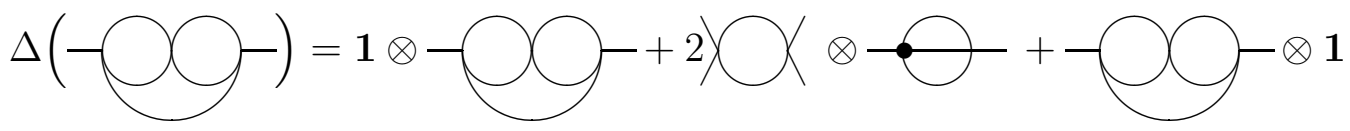

Figure 2: The "double ice cream in a cup"

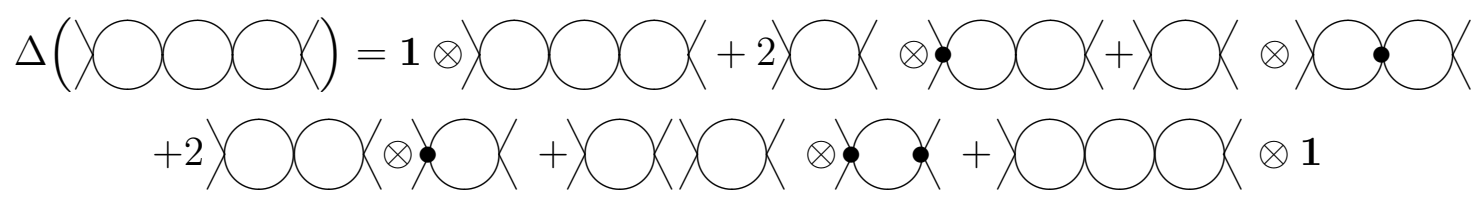

Figure 3: The "triple sweet"

That defines $H_{\Phi}$ as a bialgebra. A grading is provided by depth. A graph $\Gamma$ in the space $H_{\Phi}$ has depth $k$ (or is $k$-primitive) if

$$
P^{\otimes k+1}\left(\Delta^{k} \Gamma\right)=0 \quad \text { and } \quad P^{\otimes k}\left(\Delta^{k-1}\right) \Gamma \neq 0 .
$$

where $P$ is the projection $u \circ \varepsilon-\mathrm{id}$, whose importance was discovered "experimentally" by Figueroa and myself [81] in the context of the Hopf algebra of rooted trees [82]. 


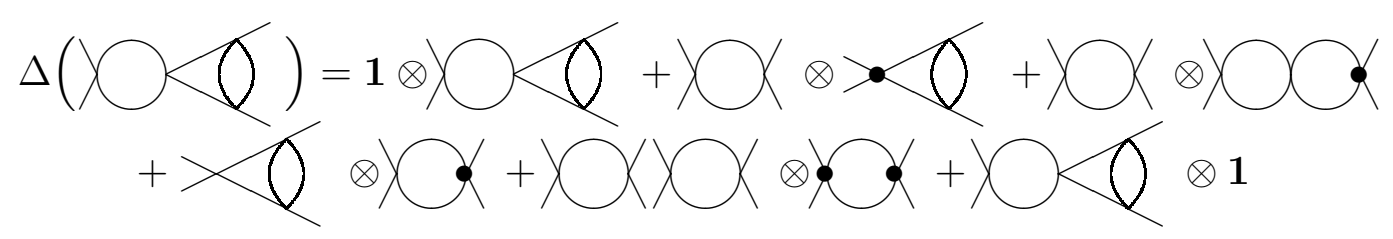

Figure 4: The "rag-doll"

The depth of any graph is finite. In our context, depth measures the maximal length of the inclusion chains of subgraphs appearing in the Bogoliubov recursion. If $\gamma \subsetneq \Gamma$ and $\Gamma$ has depth $l$, then $\gamma$ has depth $l-1$ at most. Elements of $H_{\Phi}$ without subdivergences have depth 1, obviously. This is the case of the "fish" graph in the $\varphi_{4}^{4}$ model. The "ice-cream" graph has depth 2. Of the eight four-vertex (three-loop) graphs relevant for the four-point structure of that model, five have depth 3 , two have depth 2 and one (the tetrahedron graph) has depth 1. In dimensional regularization, a graph of depth $l$ is expected to display a pole of $l$ th order.

Now $S$ can also be defined as the inverse of id $=u \circ \varepsilon-P$ for the convolution, and so (after some calculation)

$$
S\left(\Gamma_{l}\right):=\sum_{k=1}^{l} P^{* k} \Gamma_{l}=-\Gamma_{l}+\sum_{\emptyset \subsetneq \gamma \subsetneq \Gamma_{l}} S(\gamma) \Gamma_{l} / \gamma,
$$

for any graph $\Gamma_{l}$ of depth $l$.

The foregoing is my own summary of the Hopf algebra of Feynman diagrams discovered by Connes and Kreimer [83, 84. As it stands, the Hopf algebra $H_{\Phi}$ corresponds to a formal manipulation of graphs. These formulas can then be matched to expressions for numerical values. Firstly, the Feynman rules for the unrenormalized theory prescribe a linear map

$$
f: H_{\Phi} \rightarrow \mathcal{A}
$$

into some commutative algebra $\mathcal{A}$, which is multiplicative on disjoint unions: $f\left(\Gamma_{1} \Gamma_{2}\right)=$ $f\left(\Gamma_{1}\right) f\left(\Gamma_{2}\right)$. In other words, $f$ is actually a homomorphism of algebras. In dimensional regularization, $\mathcal{A}$ is an algebra of Laurent series in a complex parameter $\varepsilon$, and $\mathcal{A}$ is the direct sum of two subalgebras:

$$
\mathcal{A}=\mathcal{A}_{+} \oplus \mathcal{A}_{-} .
$$

Let $T: \mathcal{A} \rightarrow \mathcal{A}_{-}$be the projection on the second subalgebra, with $\operatorname{ker} T=\mathcal{A}_{+} \cdot \mathcal{A}_{+}$is the holomorphic subalgebra of Taylor series and $\mathcal{A}_{-}$is the subalgebra of polynomials in $1 / \varepsilon$ without constant term; the projection $T$ picks out the pole part, in a minimal subtraction scheme. Now $T$ is not a homomorphism, but the property that both its kernel and image are subalgebras is reflected in a "multiplicativity constraint": $T(a b)+T(a) T(b)=T(T(a) b)+$ $T(a T(b))$.

The equation 88a means that "the antipode delivers the counterterm": one replaces $S$ in the calculation (9) by $C$ to obtain the right hand side, before projection with $T$. From the definition of the coproduct in $H_{\Phi}$, (8b) is a convolution in $\operatorname{Hom}\left(H_{\Phi}, \mathcal{A}\right)$, namely, $R=C * f$. To show that $R$ is multiplicative, it is enough to verify that the counterterm map $C$ is 
multiplicative, since the convolution of homomorphisms is a homomorphism because $\mathcal{A}$ is commutative. In other words, one must check that (7) and (8a) are compatible; this was done by Connes and Kreimer in [83], and elaborated or exploited in subsequent papers; see [85] in this connection.

The previous discussion is logically independent of NCG, although it is true that the Hopf algebra approach to renormalization theory has been developed in close contact with the Connes-Moscovici noncommutative theory of foliations. In that theory, a foliation is described by a noncommutative algebra of functions twisted by local diffeomorphisms, and horizontal and vertical vector fields are represented on that algebra by the action of a certain Hopf algebra $H_{C M}$ which provides a way to compute a local index formula [86].

On extending the Hopf algebra $H_{\Phi}$ of graphs by incorporating operations of insertion of subgraphs, one obtains a noncommutative Hopf algebra of the $H_{C M}$ type, which gives a supplementary handle on the combinatorial structure of $H_{\Phi}$ [87.

When handled through the Hopf algebra of Feynman diagrams $H_{\Phi}$ of Connes and Kreimer, the Epstein-Glaser (EG) renormalization method provides an alternative route to the core results of renormalization theory [88, 89.

In the last few years, EG renormalization has been revived by the Hamburg group around Fredenhagen. It has also been sharpened into a more versatile tool ("T-renormalization") in my [90]. The concept of optimal scaling of the amplitudes is the soul of this improvement. A similar tack was recently taken by Hollands and Wald [91 for the precise definition of local, covariant quantum field. See also, in this respect [92, 93, 94]. The basic idea (very much in the spirit of NCG) is that, if a quantum field is thought of as a distribution on a globally hyperbolic spacetime, with values in the algebra of the Wick products, then isometric embeddings from one spacetime into another are realized as suitable homomorphisms of the fields. Then, the key property in the existence proof of time-ordered products by Hollands and Wald is the postulated, and then recursively proved, optimal rescaling of functionals of the metric $g$, up to the logarithmic terms:

$$
\lambda^{d} f_{n}\left[\lambda^{2} g\right]=f_{n}[g]+\log \lambda f_{n-1}[g]
$$

The extension to curved backgrounds of the Connes-Kreimer Hopf algebra approach to renormalization seems to hinge on the EG method.

\section{Outlook}

Will NCG live up to its promise with regard to fundamental physics?

One should not confuse the learning of a new language with the expansion of physical knowledge. A word of caution about the changing relationships between physics and mathematics is always in order. For instance, Yang and Mills found a family of theories which seemed pertinent to describe the observed phenomena. Mathematicians have translated them into fibre bundle language, and now we teach Yang-Mills theories to our students with the rigidity of formalism, so they seem completely unavoidable. However, in their present form they are suspect (at least to Mills 95] and to this reviewer), for the good reason that 
quantizing them is a nightmare. It may come to pass that eventually they be seen as an incomplete preliminary stage of a more refined physical model.

By the standards of fashion, the NCG conquest of physics is now ebbing somewhat. This ebb will surely prove temporary, since the concepts and tools of noncommutative geometry are indeed powerful and helpful in QFT. On the other hand, our ability to reformulate the $\mathrm{SM}$ as a noncommutative geometry is not, in itself, all that meaningful. A truer test for the NCG paradigm is the understanding of the fermion mass and mixing matrices.

As for NC field theory, the models available so far, while they amount to a useful mathematical laboratory for the unification of gravity and the other fundamental interactions, are still too rough. In spite of the ideology of effective field theory, it remains true that the fantastic successes of the renormalizability program, now validated for geometric bakgrounds, and of renormalizability itself as a heuristic principle in selecting physical models, tell us that the violation of locality which we expect at some level is subtler than we have been able to dream of until now. Some crucial ingredient is missing.

\section{Acknowledgements}

I am very grateful to the organizers of the 66. Tagung der Deutsche Physikalische Gesellschaft for the invitation to survey the field. Financial support for my stay at the Departamento de Física Teórica I of Universidad Complutense came from the Secretaría de Estado de Educación y Universidades of Spain. Carmelo P. Martín and Joseph C. Várilly have been helpful, and influential in shaping the opinions outlined here. Serge Lazzarini taught me the EG scheme. Special thanks are due to Roger Plymen and Henri Moscovici for sharing with me recollections of the early days of noncommutative geometry.

\section{References}

[1] A. Connes, "Spectral sequence and homology of currents for operator algebras", Tagungsbericht 42/81, Mathematisches Forschungszentrum Oberwolfach, 1981

[2] A. Connes, C. R. Acad. Sci. Paris 290 (1980) 599

[3] A. Connes, Publ. Math. IHES 39 (1985) 257

[4] J. M. Gracia-Bondía, J. C. Várilly and H. Figueroa, Elements of Noncommutative Geometry, Birkhäuser, Boston, 2001

[5] A. Connes, C. R. Acad. Sci. Paris 296 (1983) 953

[6] M. A. Rieffel, Pac. J. Math. 93 (1981) 415

[7] J. M. Gracia-Bondía, Phys. Lett. B 482 (2000) 315

[8] P. Woit, "String theory: an evaluation", physics/0102051

[9] N. Higson and J. Roe, Analytic K-Homology, Oxford University Press, Oxford, 2000 
[10] C. P. Martín, J. M. Gracia-Bondía and J. C. Várilly, Phys. Reports 294 (1998) 363

[11] J. C. Várilly and J. M. Gracia-Bondía, J. Geom. Phys. 12 (1993) 223

[12] D. Kastler and T. Schücker, Rev. Math. Phys. 8 (1996) 205.

[13] E. Alvarez, J. M. Gracia-Bondía and C. P. Martín, Phys. Lett. B 306 (1993) 55

[14] Y. Ne'eman, Phys. Lett. B 81 (1979) 190

[15] F. Scheck, "The Standard Model within noncommutative geometry: A comparison of models", hep-th/9701073

[16] M. Chaves and H. Morales, Mod. Phys. Lett. A13 (1998) 2021

[17] A. Connes, Noncommutative Geometry, Academic Press, London and San Diego, 1994

[18] E. Alvarez, J. M. Gracia-Bondía and C. P. Martín, Phys. Lett. B364 (1995) 33

[19] S. Lazzarini and T. Schücker, Phys. Lett. B510 (2001) 277

[20] H. S. Snyder, Phys. Rev. 71 (1947) 38

[21] M. M. Sheikh-Jabbari, Phys. Lett. B455 (1999) 129

[22] V. Schomerus, J. High Energy Phys. 9906 (1999) 030

[23] N. Seiberg and E. Witten, J. High Energy Phys. 9909 (1999) 032

[24] S. Kar, Nucl. Phys. B577 (2000) 171

[25] A. Connes, M. R. Douglas and A. Schwartz, J. High Energy Phys. 9802 (1998) 003

[26] M. R. Douglas and C. M. Hull, J. High Energy Phys. 9802 (1998) 008

[27] G. Landi, F. Lizzi and R. J. Szabo, Commun. Math. Phys. 206 (1999) 603

[28] J. Fröhlich and K. Gawȩdzki, "Conformal field theory and geometry of strings", in CRM Proceedings and Lecture Notes 7, CRM, Montréal, 1994, p. 57

[29] S. Doplicher, K. Fredenhagen and J. E. Roberts, Commun. Math. Phys. 172 (1995) 187

[30] B. Jurčo, P. Schupp and J. Wess, "Noncommutative line bundle and Morita equivalence", hep-th/0106110

[31] R. Jackiw and S.-Y. Pi, Phys. Lett. B534 (2002) 181

[32] R. Estrada, J. M. Gracia-Bondía and J. C. Várilly, J. Math. Phys. 30 (1989) 2789

[33] F. Lizzi, Nucl. Phys. B (Proc. Suppl.) 104 (2002) 143

[34] M. R. Douglas and N. A. Nekrasov, Rev. Mod. Phys. 73 (2002) 977 
[35] J. C. Várilly and J. M. Gracia-Bondía, Int. J. Mod. Phys. A14 (1999) 1305

[36] M. Chaichian, A. Demichev and P. Prešnajder, Nucl. Phys. B567 (2000) 360

[37] T. Filk, Phys. Lett. B376 (1996) 53

[38] A. González-Arroyo and C. P. Korthals-Altes, Phys. Lett. B131 (1983) 396

[39] C. P. Martín and D. Sánchez-Ruiz, Phys. Rev. Lett. 83 (1999) 476

[40] T. Krajewski and R. Wulkenhaar, Int. J. Mod. Phys. A15 (2000) 1011

[41] J. A. Harvey, "Komaba lectures on noncommutative solitons and D-branes", hep-th/0102076

[42] J. M. Gracia-Bondía, Contemp. Math. 134 (1992) 93

[43] J. M. Gracia-Bondía and J. C. Várilly, J. Math. Phys. 29 (1988) 869

[44] J. C. Várilly and J. M. Gracia-Bondía, J. Math. Phys. 29 (1988) 880

[45] M. Chaichian, P. Prešnajder, M. M. Sheikh-Jabbari and A. Tureanu, Phys. Lett. B526 (2002) 132

[46] S. Terashima, Phys. Lett. B482 (2000) 276

[47] C. P. Korthals-Altes, in Progress in Gauge Field Theory (Cargèse lectures), G. 't Hooft et al, eds., Plenum, 1985, p. 425

[48] C. P. Martín and D. Sánchez-Ruiz, Nucl. Phys. B598 (2001) 348

[49] H. O. Girotti, M. Gomes, V. O. Rivelles and A. J. da Silva, Nucl. Phys. B587 (2000) 299

[50] S. Paban, S. Sethi and M. Stern, J. High Energy Phys. 0203 (2002) 012

[51] R. Wulkenhaar, J. High Energy Phys. 0203 (2002) 024

[52] J. Gomis and T. Mehen, Nucl. Phys. B591 (2000) 265

[53] L. Alvarez-Gaumé, J. L. F. Barbón and R. Zwicky, J. High Energy Phys. 0105 (2001) 057

[54] D. Bahns, S. Doplicher, K. Fredenhagen and G. Piacitelli, Phys. Lett. B533 (2002) 178

[55] C. Rim and J. H. Yee, "Unitarity in space-time noncommutative field theories", hep-th/0205193

[56] Y. Liao and K. Sibold, Eur. Phys. J. C 25 (2002) 469

[57] D. Bahns, private communication 
[58] H. Dorn, Fortschr. Phys. 50 (2002) 884

[59] J. M. Gracia-Bondía and C. P. Martín, Phys. Lett. B479 (2000) 321

[60] K. Intriligator and J. Kumar, Nucl. Phys. B620 (2002) 315

[61] C. P. Martín, J. Phys. A34 (2001) 9037

[62] A. Armoni, E. Lopez and S. Theisen, J. High Energy Phys. 0206 (2002) 050

[63] K. Morita, "Connes' gauge theory on noncommutative spacetimes", hep-th/0011080

[64] M. Chaichian, P. Prešnajder, M. M. Sheikh-Jabbari and A. Tureanu, "Noncommutative Standard Model: model building", hep-th/0107055

[65] X. Calmet, B. Jurčo, P. Schupp, J. Wess and M. Wohlgenannt, Eur. Phys. J. C23 (2002) 363

[66] J. L. Hewett, F. J. Petriello and T. G. Rizzo, Phys. Rev. D64 (2001) 075012

[67] C.-S. Chu, B. R. Greene and G. Shiu, Mod. Phys. Lett. A16 (2001) 2231

[68] F. Lizzi, G. Mangano, G. Miele and M. Peloso, J. High Energy Phys. 0206 (2002) 049

[69] A. H. Chamseddine and A. Connes, Commun. Math. Phys. 186 (1997) 731

[70] R. Estrada, J. M. Gracia-Bondía and J. C. Várilly, Commun. Math. Phys. 191 (1998) 219

[71] H. Figueroa, J. M. Gracia-Bondía, F. Lizzi and J. C. Várilly, J. Geom. Phys. 26 (1998) 329

[72] J. M. Gracia-Bondía, F. Lizzi, G. Marmo and P. Vitale, J. High Energy Phys. 0204 (2002) 026

[73] A. Connes, J. Math. Phys. 41 (2000) 3832

[74] A. Connes and G. Landi, Commun. Math. Phys. 221 (2001) 141

[75] J. C. Várilly, Commun. Math. Phys. 221 (2001) 511

[76] A. Sitarz, Lett. Math. Phys. 55 (2001) 127

[77] A. Connes and M. Dubois-Violette, Commun. Math. Phys. 230 (2002) 539

[78] E. Alvarez, "Some general problems in quantum gravity", in Quantum Gravity and Cosmology, World Scientific, Singapore, 1992

[79] A. Connes, Commun. Math. Phys. 182 (1996) 155

[80] A. Connes, private communication 
[81] H. Figueroa and J. M. Gracia-Bondía, Mod. Phys. Lett. A16 (2001) 1427

[82] A. Connes and D. Kreimer, Commun. Math. Phys. 199 (1998) 203

[83] A. Connes and D. Kreimer, Commun. Math. Phys. 210 (2000) 249

[84] A. Connes and D. Kreimer, Commun. Math. Phys. 216 (2001) 215

[85] F. Girelli, P. Martinetti and T. Krajewski, Mod. Phys. Lett. A16 (2001) 299

[86] A. Connes and H. Moscovici, Commun. Math. Phys. 198 (1998) 198

[87] A. Connes and D. Kreimer, Ann. Henri Poincaré 3 (2002) 411

[88] G. Pinter, Ann. Phys. (Leipzig) 10 (2001) 333

[89] G. Pinter, Lett. Math. Phys. 54 (2000) 227

[90] J. M. Gracia-Bondía, "Improved Epstein-Glaser renormalization in coordinate space I. Euclidean framework", hep-th/0202023

[91] S. Hollands and R. M. Wald, Commun. Math. Phys. 231 (2002) 309

[92] R. Brunetti, K. Fredenhagen and R. Verch, "The generally covariant locality principle - A new paradigm for local quantum field theory", math-ph/0112041

[93] W. Junker and E. Schrohe, "Adiabatic vacuum states on general spacetime manifolds: definition, construction, and physical properties", Potsdam, 2001

[94] D. R. Grigore, Class. Quant. Grav. 17 (2000) 319

[95] R. Mills, "Beauty and truth", in Chen Ning Yang: A Great Physicist of the Twentieth Century, C. S. Liu and S.-T. Yau, eds., International Press, Cambridge, MA, 1995, p. 199 